\title{
Phase states of nanocrystalline ferroelectric ceramics and their dielectric properties
}

\author{
A. G. Zembilgotov, ${ }^{\text {a) }}$ N. A. Pertsev, ${ }^{\text {b) }}$ and R. Waser ${ }^{\text {() }}$ \\ Institut für Werkstoffe der Elektrotechnik, Rheinisch Westfälische Technische Hochschule (RWTH) Aachen \\ University of Technology, Aachen D-52056, Germany
}

(Received 12 July 2004; accepted 4 April 2005; published online 27 May 2005)

\begin{abstract}
Using a nonlinear thermodynamic theory, we describe equilibrium polarization states and the macroscopic dielectric response of nanocrystalline ferroelectric ceramics with single-domain grains. The elastic clamping of individual crystallites by the surrounding material is explicitly taken into account via the introduction of a specific thermodynamic potential. Aggregate material properties are calculated with the aid of an iterative procedure based on the method of effective medium. The numerical calculations, performed for unpolarized $\mathrm{BaTiO}_{3}$ and $\mathrm{Pb}\left(\mathrm{Zr}_{1-x} \mathrm{Ti}_{x}\right) \mathrm{O}_{3}$ ceramics, demonstrate that the equilibrium phase states of nanocrystalline ceramics may differ drastically from those of single crystals and coarse-grained materials. Remarkably, the theory predicts the coexistence of rhombohedral and tetragonal crystallites in nanocrystalline $\mathrm{Pb}\left(\mathrm{Zr}_{1-x} \mathrm{Ti}_{x}\right) \mathrm{O}_{3}$ ceramics in a wide range of compositions and temperatures. For $\mathrm{BaTiO}_{3}$ ceramics, a mixture of rhombohedral and orthorhombic crystallites is found to be the energetically most favorable state at room temperature. The calculations also show that the dielectric properties of nanocrystalline ferroelectric ceramics may be very different from those of conventional materials due to the elastic clamping of single-domain crystallites. (C) 2005 American Institute of Physics. [DOI: 10.1063/1.1924875]
\end{abstract}

\section{INTRODUCTION}

Polycrystalline ferroelectric materials are widely employed in modern microelectronics. ${ }^{1}$ Former experimental investigations of these materials demonstrated that the dielectric response and domain structure of ferroelectric ceramics depend on the grain size. ${ }^{2-6}$ A further reduction of crystallite dimensions down to the nanoscale may lead to the appearance of unusual physical properties as compared to those of conventional polycrystals. Some data on the microstructure and behavior of nanocrystalline powders, ceramics, and thin films of $\mathrm{BaTiO}_{3}, \mathrm{PbTiO}_{3}$, and $\mathrm{Pb}\left(\mathrm{Zr}_{1-x} \mathrm{Ti}_{x}\right) \mathrm{O}_{3}$ have been already obtained, ${ }^{7-13}$ and the experimental results indicate even the disappearance of ferroelectricity below a critical crystal size.

Specific physical properties of nanocrystalline ferroelectrics may arise from several different reasons. These include the intrinsic size effect on ferroelectricity, ${ }^{14-16}$ the influence of depolarizing field, ${ }^{17,18}$ the surface tension effect, ${ }^{8}$ and the surface bond contraction. ${ }^{19}$ In ceramic materials, where individual crystallites are surrounded by a ferroelectric medium, the mechanical effect caused by the elastic three-dimensional (3D) clamping of crystallites may play an important role. Indeed, below some critical grain size, the twinning of crystallites, which strongly reduces internal stresses in coarsegrained ceramics, becomes energetically unfavorable. ${ }^{5} \mathrm{Ow}$ ing to the mechanical grain/grain interactions, high

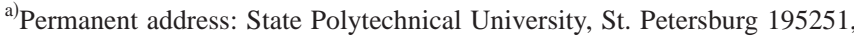
Russia; electronic mail: az@ domain.ioffe.rssi.ru

${ }^{b)}$ Permanent address: A. F. Ioffe Physico-Technical Institute, Russian Academy of Sciences, St. Petersburg 194021, Russia; electronic mail: pertsev@domain.ioffe.rssi.ru

${ }^{c}$ Also at Institut für Festkörperforschung, Forschungszentrum Jülich, Jülich D-52425, Germany.
}

mechanical stresses appear in single-domain crystallites below the ferroelectric phase transition, which alter their physical properties. As shown by Buessem et al. ${ }^{20}$ the presence of such stresses may explain the observed strong increase of permittivity in fine-grained $\mathrm{BaTiO}_{3}$ ceramics. In their classical paper, a simplified model of a tetragonal crystallite under a given constant stress was used to calculate the dielectric response. ${ }^{20}$

For the correct theoretical description of ferroelectricity in elastically clamped crystallites, the mechanical boundary conditions existing on the grain boundaries must be properly taken into account. Since clamped crystallites in dense ceramics are neither under a constant stress nor under a constant strain, a specific thermodynamic potential must be introduced to describe the effect of the elastic 3D clamping on homogeneously polarized ferroelectric crystallites. ${ }^{21,22}$ The minimization of this potential $\widetilde{G}$ makes it possible to determine the equilibrium thermodynamic state of a crystallite embedded in a homogeneous linear medium with given elastic stiffnesses. In the case of ceramics, however, these stiffnesses must be regarded as unknown quantities because they represent the aggregate material properties on the macroscopic level. Therefore, the determination of the phase states and physical properties of ferroelectric ceramics calls for the use of a self-consistent scheme.

Up to now, this scheme, also called the effective medium approach, has been applied only to the calculations of aggregate material constants, which were performed in the linear approximation. ${ }^{23-30}$ In the present paper, we combine the method of effective medium with the nonlinear thermodynamic theory of ferroelectrics ${ }^{31-33}$ to calculate the actual polarization states and material constants of $\mathrm{BaTiO}_{3}$ and $\mathrm{Pb}\left(\mathrm{Zr}_{1-x} \mathrm{Ti}_{x}\right) \mathrm{O}_{3}$ ceramics with single-domain grains. Since 
this nonlinear approach takes into account the polarization changes caused by the elastic 3D clamping of crystallites, it enables the correct determination of the physical properties of ferroelectric ceramics.

In Sec. II, a general expression is derived for the thermodynamic potential $\widetilde{G}$ of a spherical ferroelectric crystallite (inclusion) clamped by a linear elastic medium (matrix). In contrast to the previous treatment of the problem, ${ }^{22}$ we allow for the presence of inelastic (spontaneous) strains in the matrix. The influence of external mechanical and electric fields on the thermodynamic state of a ferroelectric inclusion is considered in Sec. III. An iterative procedure is also described here, which makes it possible to calculate the aggregate material constants of nanocrystalline ferroelectric ceramics. The results of our numerical calculations performed for unpolarized $\mathrm{BaTiO}_{3}$ and $\mathrm{Pb}\left(\mathrm{Zr}_{1-x} \mathrm{Ti}_{x}\right) \mathrm{O}_{3}$ ceramics are reported in Sec. IV. We describe the stability ranges of various phase states in these ceramics and their dielectric properties and compare our theoretical predictions with available experimental data. Finally, main conclusions of this study are formulated in Sec. V.

\section{DETERMINATION OF EQUILIBRIUM POLARIZATION STATE OF A FERROELECTRIC INCLUSION}

We start with the development of a thermodynamic formalism that enables us to determine the equilibrium phase state of a single-domain ferroelectric crystallite (inclusion) embedded into a linear elastic medium (matrix). Owing to the finite conductivity of perovskite ferroelectrics, in this section we neglect the influence of internal electric fields caused by the presence of electric polarization $\mathbf{P}$ inside the inclusion. For simplicity, we shall assume the inclusion to have a spherical shape and the matrix to be homogeneous, nonpiezoelectric, and isotropic (this approximation is sufficient for the modeling of unpolarized bulk ceramics). In contrast to the previous treatment of the problem, ${ }^{22}$ the matrix is allowed to possess uniform inelastic strains $S_{i j}^{0 m}(i, j=1,2,3)$. When $S_{i j}^{0 m}=0$ and the crystallite is in a paraelectric phase, the inclusion/matrix system is taken to be free of internal mechanical stresses $\sigma_{i j}$.

The stable thermodynamic states of an elastically clamped ferroelectric crystallite correspond to the minima of the modified thermodynamic potential $\widetilde{G}$ introduced in Ref. 22. Generalizing the basic relation for $\widetilde{G}$ derived in Ref. 22, we obtain the following formula:

$$
\widetilde{G}=F-\frac{1}{2}\left(S_{i j}-S_{i j}^{0 m}\right) \sigma_{i j},
$$

where $F, S_{i j}$, and $\sigma_{i j}$ are the (homogeneous) Helmholtz freeenergy density, total strain, and stress inside the ferroelectric inclusion, respectively. For our purposes, it is convenient to replace $F$ in Eq. (1) by the Gibbs free-energy density $G$ via the inverse Legendre transformation $F=G+S_{i j} \sigma_{i j}$. Therefore, the modified thermodynamic potential may be written as

$$
\widetilde{G}=G+\frac{1}{2} S_{i j} \sigma_{i j}+\frac{1}{2} S_{i j}^{0 m} \sigma_{i j} .
$$

Using an explicit expression for the Gibbs energy $G$ of a ferroelectric given in Ref. 34, we derive
$\widetilde{G}=G_{0}\left(P_{i}\right)-\frac{1}{2} s_{i j k l}^{P} \sigma_{i j} \sigma_{k l}-Q_{i j k l} \sigma_{i j} P_{k} P_{l}+\frac{1}{2} S_{i j} \sigma_{i j}+\frac{1}{2} S_{i j}^{0 m} \sigma_{i j}$,

where $s_{i j k l}^{P}$ are the elastic compliances at constant polarization, $Q_{i j k l}$ are the electrostrictive constants in full polarization notation, and $G_{0}\left(P_{i}\right)$ is the polynomial in terms of polarization components $P_{i}$ inside the inclusion. In the $P^{6}$ approximation, which is necessary to describe ferroelectrics with the first-order phase transition, $G_{0}\left(P_{i}\right)$ has the form ${ }^{34}$

$$
\begin{aligned}
G_{0}\left(P_{i}\right)= & \alpha_{1}\left(P_{1}^{2}+P_{2}^{2}+P_{3}^{2}\right)+\alpha_{11}\left(P_{1}^{4}+P_{2}^{4}+P_{3}^{4}\right) \\
& +\alpha_{12}\left(P_{1}^{2} P_{2}^{2}+P_{2}^{2} P_{3}^{2}+P_{1}^{2} P_{3}^{2}\right) \\
& +\alpha_{111}\left(P_{1}^{6}+P_{2}^{6}+P_{3}^{6}\right)+\alpha_{112}\left[P_{1}^{4}\left(P_{2}^{2}+P_{3}^{2}\right)\right. \\
& \left.+P_{2}^{4}\left(P_{1}^{2}+P_{3}^{2}\right)+P_{3}^{4}\left(P_{1}^{2}+P_{2}^{2}\right)\right]+\alpha_{123} P_{1}^{2} P_{2}^{2} P_{3}^{2},
\end{aligned}
$$

where $\alpha_{1}, \alpha_{i j}$, and $\alpha_{i j k}$ are the dielectric stiffness and higherorder stiffness coefficients at constant stress.

To find the equilibrium thermodynamic state of a ferroelectric inclusion, we should express the potential $\widetilde{G}$ solely in terms of the polarization components $P_{i}$. Mechanical stresses $\sigma_{i j}$ can be excluded from Eq. (3) using the nonlinear equation of state of a ferroelectric crystal

$$
\sigma_{i j}=\frac{\partial F}{\partial S_{i j}}=c_{i j k l}^{P} S_{k l}-c_{i j m n}^{P} Q_{m n k l} P_{k} P_{l},
$$

which may be obtained by differentiating the Helmholtz free energy $\left(c_{i j k l}^{P}\right.$ are the elastic stiffnesses of the ferroelectric inclusion at constant polarization). In turn, relationships between strains $S_{k l}$ and polarization components $P_{i}$ should be found from the condition of mechanical equilibrium of the inclusion/matrix system. This problem can be solved by the generalization of Eshelby's equivalent inclusion technique. $^{30,35}$ Using this formalism and performing the algebraic manipulations similar to those described in Ref. 22, one can obtain the relationship

$$
\begin{aligned}
S_{k l}= & B_{(k l) m n} c_{m n p q}^{*} \tilde{s}_{p q r s} c_{r s i j}^{P} Q_{i j t u} P_{t} P_{u} \\
& -B_{(k l) m n} c_{m n p q}^{*} \tilde{s}_{p q r s} c_{r s u v}^{P} S_{u v}^{0 m}+S_{k l}^{0 m} .
\end{aligned}
$$

Here $c_{m n p q}^{*}$ are the elastic stiffnesses of the matrix, $B_{(k l) m n}$ $=(1 / 2)\left(B_{k l m n}+B_{l k m n}\right)$, and $B_{k l m n}$ is the basic matrix of a homogeneous inclusion/matrix system given by

$$
B_{k l m n}=\frac{1}{4 \pi} \int_{\Omega} \frac{z_{k}(z z)_{l m}^{-1} z_{n}}{\left(z_{1}^{2}+z_{2}^{2}+z_{3}^{2}\right)^{3 / 2}} d \Omega,
$$

where the integration is carried out over the surface $\Omega$ of the unit sphere, $z_{i}$ are the components of the unit vector $\mathbf{z}$, and $(z z)_{l m}^{-1}$ is the inverse of the real symmetric $(3 \times 3)$ matrix $(z z)_{k l}=z_{i} c_{i l k j}^{*} z_{j}$. The tensor $\tilde{s}_{u v i j}$ depends only on the elastic constants $c_{i j k l}^{*}$ and $c_{i j k l}^{P}$, being defined by the system of equations $\left(\delta_{k l}\right.$ is the Kronecker delta)

$$
\tilde{s}_{u v i j}\left[\left(c_{i j k l}^{*}-c_{i j k l}^{P}\right) B_{(k l) m n} c_{m n p q}^{*}-c_{i j p q}^{*}\right]=-\delta_{u p} \delta_{v q} .
$$

It should be noted that Eq. (6) for the inclusion strains $S_{k l}$ differs from the corresponding formula derived in Ref. 22 by the presence of two additional terms depending on the matrix inelastic strains $S_{k l}^{0 m}$. 
Substituting Eqs. (5) and (6) into Eq. (3), after some algebraic manipulation, we find the thermodynamic potential $\widetilde{G}$ as a function of the polarization components $P_{i}$ only. The result may be written as

$$
\begin{aligned}
\widetilde{G}= & G_{0}\left(P_{i}\right)-\frac{1}{2} B_{(m n) i j} c_{i j k l}^{*} \widetilde{s}_{k l r s} \lambda_{r s p q} \lambda_{m n t u} P_{t} P_{u} P_{p} P_{q} \\
& +\frac{1}{2} c_{i j k l}^{P} Q_{k l p q} Q_{i j t u} P_{t} P_{u} P_{p} P_{q}-c_{k l m n}^{P} Q_{m n p q} S_{k l}^{M e} P_{p} P_{q} \\
& +\frac{1}{2} S_{u v}^{0 m} c_{u v m n}^{P} S_{m n}^{M e},
\end{aligned}
$$

where $\lambda_{i j k l}=-c_{i j m n}^{P} Q_{m n k l}$ and

$$
S_{k l}^{M e}=S_{k l}^{0 m}-B_{(k l) m n} c_{m n p q}^{*} \tilde{S}_{p q r s} c_{r s u v}^{P} S_{u v}^{0 m} .
$$

Expressions (9) and (10) enable us to find the stable polarization state of a ferroelectric inclusion via the minimization of the potential $\widetilde{G}$ with respect to three variables, $P_{1}, P_{2}$, and $P_{3}$. If several local minima exist, the equilibrium state of inclusion/matrix system corresponds to the deepest minimum of $\widetilde{G}$.

\section{SELF-CONSISTENT CALCULATION OF EFFECTIVE MATERIAL CONSTANTS}

To calculate material constants of a ferroelectric ceramic, we employ the method of effective medium ${ }^{23-30}$ together with an iterative procedure. This approach deals with the model material system, which involves a single representative ferroelectric crystallite (inclusion) surrounded by a homogeneous elastic medium (matrix). On each iteration, certain elastic stiffnesses $c_{i j k l}^{*}$, dielectric constants $\varepsilon_{i j}^{*}$, and inelastic (spontaneous) strains $S_{i j}^{0 m}$ are assigned to the matrix. In the absence of applied electric and mechanical fields, the polarization state of a spherical single-domain inclusion, defined by the equilibrium polarization components $P_{i}^{g}$, can be found as described in Sec. II. The substitution of $P_{i}^{g}$ into Eq. (6) enables us to calculate the total strains $S_{k l}^{g}$ inside the inclusion. Mechanical stresses $\sigma_{k l}^{g}$ existing in the inclusion can be computed then by substituting $P_{i}^{g}$ and $S_{k l}^{g}$ into Eq. (5). It should be recalled that we assumed the internal electric field to be zero in equilibrium $\left(E^{g}=0\right)$ because the polarization charges are expected to be largely compensated by free charges due to the finite conductivity of ferroelectric ceramics. In what follows, however, the material system is treated as a perfect insulator since the charge carriers remain practically immobile during the dielectric measurements.

Suppose now that the inhomogeneous inclusion/matrix system under consideration is subjected to a uniform strain field $S_{m n}^{a}$ and electric field $E_{n}^{a}$ at large distances from the ferroelectric inclusion $V$. These external fields alter the polarization components, total strain, and stress inside the inclusion, which may be written as $P_{i}=P_{i}^{g}+\Delta P_{i}, S_{m n}=S_{m n}^{g}$ $+\Delta S_{m n}$, and $\sigma_{i j}=\sigma_{i j}^{g}+\Delta \sigma_{i j}$. An electric field $E_{n}=E_{n}^{g}+\Delta E_{n}$ $=\Delta E_{n}$ also appears in the inclusion, being different from $E_{n}^{a}$. Since $P_{i}, S_{m n}$, and $\sigma_{i j}$ remain uniform inside $V, 36,37$ we can use Eshelby's equivalent inclusion technique ${ }^{30,35}$ to calculate the perturbations $\Delta P_{i}, \Delta S_{m n}, \Delta \sigma_{i j}$ and the field $\Delta E_{n}$.

To solve the problem, we introduce another material system, where the ferroelectric inclusion is replaced by a sphere $V^{*}$ having linear elastic and dielectric properties and exactly the same material constants $c_{i j k l}^{*}$ and $\varepsilon_{i j}^{*}$ as the surrounding matrix. This "homogeneous" inclusion $V^{*}$ is assumed to undergo a uniform transformation (inelastic) strain $S_{k l}^{0 x}$ and acquire a uniform permanent polarization $P_{i}^{0 x}$ in the absence of mechanical stresses and depolarization fields. Since the medium is linear everywhere, the total strain $\Delta S_{m n}^{*}$ and electric field $\Delta E_{\eta}^{*}$ inside the transformed sphere $V^{*}$ are given by the relations ${ }^{30}$

$$
\begin{aligned}
& \Delta S_{m n}^{*}=S_{m n}^{a}+B_{(n m) j i} c_{i j k l}^{*} S_{k l}^{0 x}, \\
& \Delta E_{n}^{*}=E_{n}^{a}+B_{n 44 i} P_{i}^{0 x},
\end{aligned}
$$

where $B_{n 44 i}$ is the matrix defined by an expression, which differs from Eq. (7) by the replacement of $(z z)_{l m}^{-1}$ by $(z z)_{44}^{-1}$ with $(z z)_{44}=-z_{i} \varepsilon_{i k}^{*} z_{k}$.

For the stress $\Delta \sigma_{i j}^{*}$ and polarization $\Delta P_{n}^{*}$ existing in the inclusion $V^{*}$, we have $\left(\varepsilon_{0}\right.$ is the permittivity of the vacuum)

$$
\begin{aligned}
& \Delta \sigma_{i j}^{*}=c_{i j k l}^{*}\left(\Delta S_{k l}^{*}-S_{k l}^{0 x}\right), \\
& \Delta P_{n}^{*}=P_{n}^{0 x}+\varepsilon_{n i}^{*} \Delta E_{i}^{*}-\varepsilon_{0} \Delta E_{n}^{*} .
\end{aligned}
$$

To ensure the equivalence of the introduced inclusion to the perturbation of the actual one, the following relationships must hold:

$$
\begin{aligned}
& \Delta \sigma_{i j}^{*}=\Delta \sigma_{i j}, \\
& \Delta P_{n}^{*}=\Delta P_{n}, \\
& \Delta S_{m n}^{*}=\Delta S_{m n}, \\
& \Delta E_{n}^{*}=\Delta E_{n} .
\end{aligned}
$$

At the same time, the strain perturbation $\Delta S_{m n}$ and the electric field $\Delta E_{n}$ in the ferroelectric inclusion may be expressed in terms of the derivatives of the Gibbs energy $G$ as

$$
\begin{aligned}
& \Delta S_{m n}=-g_{m n}^{S}\left(P_{i}^{g}+\Delta P_{i}, \sigma_{k l}^{g}+\Delta \sigma_{k l}\right)-S_{m n}^{g}, \\
& \Delta E_{n}=g_{n}^{E}\left(P_{i}^{g}+\Delta P_{i}, \sigma_{k l}^{g}+\Delta \sigma_{k l}\right),
\end{aligned}
$$

where

$$
\begin{aligned}
& g_{m n}^{S}\left(P_{i}, \sigma_{k l}\right)=\frac{\partial G\left(P_{i}, \sigma_{k l}\right)}{\partial \sigma_{m n}}, \\
& g_{n}^{E}\left(P_{i}, \sigma_{k l}\right)=\frac{\partial G\left(P_{i}, \sigma_{k l}\right)}{\partial P_{n}} .
\end{aligned}
$$

Using the equivalence conditions (15) and (16), we can substitute $\Delta \sigma_{i j}^{*}$ for $\Delta \sigma_{i j}$ and $\Delta P_{n}^{*}$ for $\Delta P_{n}$ in the right-hand sides of Eqs. (19) and (20). Then, in accordance with the equivalence relations (17) and (18), the right-hand sides of Eqs. (11) and (12) may be equated with the right-hand sides of Eqs. (19) and (20), respectively. This procedure yields

$S_{m n}^{a}+B_{(n m) j i} c_{i j k l}^{*} S_{k l}^{0 x}=-g_{m n}^{S}\left(P_{i}^{g}+\Delta P_{i}^{*}, \sigma_{k l}^{g}+\Delta \sigma_{k l}^{*}\right)-S_{m n}^{g}$, 


$$
E_{n}^{a}+B_{n 44 i} P_{i}^{0 x}=g_{n}^{E}\left(P_{i}^{g}+\Delta P_{i}^{*}, \sigma_{k l}^{g}+\Delta \sigma_{k l}^{*}\right)
$$

Excluding the stress $\Delta \sigma_{k l}^{*}$ and polarization $\Delta P_{i}^{*}$ from Eqs. (23) and (24) with the aid of Eqs. (13) and (14), we finally obtain

$$
\begin{aligned}
S_{m n}^{a}+B_{(n m) j i} c_{i j k l}^{*} S_{k l}^{0 x}= & -g_{m n}^{S}\left[P_{i}^{g}+P_{i}^{0 x}+\varepsilon_{i r}^{*} \Delta E_{r}^{*}-\varepsilon_{0} \Delta E_{i}^{*}, \sigma_{k l}^{g}\right. \\
& \left.+c_{k l u v}^{*}\left(\Delta S_{u v}^{*}-S_{u v}^{0 x}\right)\right]-S_{m n}^{g},
\end{aligned}
$$

$$
\begin{aligned}
E_{n}^{a}+B_{n 44 i} P_{i}^{0 x}= & g_{n}^{E}\left[P_{i}^{g}+P_{i}^{0 x}+\varepsilon_{i r}^{*} \Delta E_{r}^{*}-\varepsilon_{0} \Delta E_{i}^{*}, \sigma_{k l}^{g}\right. \\
& \left.+c_{k l u v}^{*}\left(\Delta S_{u v}^{*}-S_{u v}^{0 x}\right)\right] .
\end{aligned}
$$

Since the derivatives of the Gibbs free energy $G$ can be found in an explicit form by differentiating the well-known expression for $G,{ }^{34}$ the relations (25) and (26) at given values of $S_{m n}^{a}, E_{n}^{a}, S_{m n}^{g}$, and $P_{i}^{g}$ constitute a system of nine simultaneous equations in nine unknowns, $S_{u v}^{0 x}$ and $P_{i}^{0 x}$. This system can be solved numerically, thus enabling us to calculate $S_{u v}^{0 x}$, $P_{i}^{0 x}$ and then $\Delta S_{m n}^{*}, \Delta E_{n}^{*}, \Delta \sigma_{i j}^{*}$, and $\Delta P_{n}^{*}$ from Eqs. (11)-(14). Accordingly, the sought perturbations $\Delta P_{i}, \Delta S_{m n}, \Delta \sigma_{i j}$, and $\Delta E_{n}$ of the inclusion state, which are induced by the applied strain $S_{m n}^{a}$ and electric field $E_{n}^{a}$, can be determined by means of numerical calculations.

This result enables us to proceed to the calculation of the effective elastic and dielectric constants of a ferroelectric ceramic. To that end, we introduce two Cartesian coordinate systems: the sample (ceramic) system $(x, y, z)$ associated with the matrix and the crystallographic coordinate system $\left(x^{\prime}, y^{\prime}, z^{\prime}\right)$ of the crystallite (inclusion) in the paraelectric state. Orientation of the crystallographic system $\left(x^{\prime}, y^{\prime}, z^{\prime}\right)$ relative to the ceramic reference frame $(x, y, z)$ may be defined by the Euler angles $\varphi, \psi$, and $\theta$. While the effective medium is isotropic and nonpiezoelectric in our case, each individual ferroelectric crystallite is anisotropic. Therefore, the stress perturbation $\Delta \sigma_{i j}$ and electric induction $\Delta D_{n}$ $=\Delta P_{n}+\varepsilon_{0} \Delta E_{n}$, which are induced in a representative crystallite by the external strain $S_{m n}^{a}$ and electric field $E_{n}^{a}$ set in the sample frame $(x, y, z)$, depend on the spatial orientation $(\varphi, \psi, \theta)$ of its crystal lattice. In an untextured ceramic, all lattice orientations of crystallites in the paraelectric state are equally probable. For the normalized distribution function $f(\varphi, \psi, \theta)$ of the Euler angles, in this situation we have $f(\varphi, \psi, \theta)=1 /\left(8 \pi^{2}\right)$. Hence the average values $\left\langle S_{m n}^{g}\right\rangle,\left\langle\Delta \sigma_{i j}\right\rangle$, and $\left\langle\Delta D_{n}\right\rangle$ of the total strains $S_{m n}^{g}(\varphi, \psi, \theta)$, stresses $\Delta \sigma_{i j}(\varphi, \psi, \theta)$, and electric induction $\Delta D_{n}(\varphi, \psi, \theta)$ in an ensemble of crystallites can be found in the ceramic reference frame as

$$
\begin{aligned}
& \left\langle S_{m n}^{g}\right\rangle=\frac{1}{8 \pi^{2}} \int_{0}^{2 \pi} d \varphi \int_{0}^{2 \pi} d \psi \int_{0}^{\pi} S_{m n}^{g}(\varphi, \psi, \theta) \sin \theta d \theta, \\
& \left\langle\Delta \sigma_{i j}\right\rangle=\frac{1}{8 \pi^{2}} \int_{0}^{2 \pi} d \varphi \int_{0}^{2 \pi} d \psi \int_{0}^{\pi} \Delta \sigma_{i j}(\varphi, \psi, \theta) \sin \theta d \theta,
\end{aligned}
$$

$$
\left\langle\Delta D_{n}\right\rangle=\frac{1}{8 \pi^{2}} \int_{0}^{2 \pi} d \varphi \int_{0}^{2 \pi} d \psi \int_{0}^{\pi} \Delta D_{n}(\varphi, \psi, \theta) \sin \theta d \theta .
$$

Calculating numerically the dependences of $\left\langle\Delta \sigma_{i j}\right\rangle$ and $\left\langle\Delta D_{n}\right\rangle$ on the applied fields $S_{m n}^{a}$ and $E_{n}^{a}$, we can evaluate the macroscopic material constants of the aggregate of crystallites as

$$
\begin{aligned}
& \left\langle c_{i j m n}\right\rangle=\frac{\partial\left\langle\Delta \sigma_{i j}\right\rangle}{\partial S_{m n}^{a}}, \\
& \left\langle\varepsilon_{i n}\right\rangle=\frac{\partial\left\langle\Delta D_{i}\right\rangle}{\partial E_{n}^{a}} .
\end{aligned}
$$

To make the solution of the problem self-consistent, these constants must coincide with the matrix constants $c_{i j k l}^{*}$ and $\varepsilon_{i j}^{*}$, and the average spontaneous strain $\left\langle S_{m n}^{g}\right\rangle$ must be equal to the matrix spontaneous strain $S_{m n}^{0 m}$. From the mathematical point of view, the introduction of these conditions is equivalent to the addition of several new equations to the system (25) and (26), with the corresponding increase of the number of variables.

Since the resulting system of nonlinear equations is transcendental and so cannot be solved analytically, we propose the following iterative procedure to obtain the solution numerically:

Consider first a mechanically free ferroelectric crystallite in a single-domain state. Using the Gibbs freeenergy function $G$ of a ferroelectric, calculate the equilibrium polarization components $P_{i^{\prime}}$, spontaneous strains $S_{i^{\prime} j^{\prime}}^{0}=Q_{i^{\prime} j^{\prime} k^{\prime} l^{\prime}} P_{k^{\prime}} P_{l^{\prime}}$, and the small-signal elastic and dielectric constants $c_{i^{\prime} j^{\prime} k^{\prime} l^{\prime}}^{P}$ and $\varepsilon_{i^{\prime} j^{\prime}}^{\sigma}$ in the crystallographic reference frame $\left(x^{\prime}, y^{\prime}, z^{\prime}\right)$. Then transform these tensors to the ceramic coordinate system $(x, y, z)$, using the Euler angles $(\varphi, \psi, \theta)$ which define the relative orientation of these reference frames. Averaging over all possible lattice orientations $(\varphi, \psi, \theta)$ with the aid of integral relations similar to Eq. (27), compute mean values of the spontaneous strains $S_{i j}^{0}$ and material constants $c_{i j k l}^{P}$ and $\varepsilon_{i j}^{\sigma}$ for the considered ensemble of crystallites. Employ these values as a first approximation for the matrix parameters $S_{i j}^{0 m}, c_{i j k l}^{*}$, and $\varepsilon_{i j}^{*}$.

(ii) Calculate numerically the basic matrices $B_{k l m n}$ and $B_{n 44 i}$ by substituting the above approximate values of the material constants $c_{i j k l}^{*}$ and $\varepsilon_{i j}^{*}$ into Eq. (7).

(iii) Determine the equilibrium polarization components $P_{i}^{g}$ and total strains $S_{m n}^{g}$ in a representative ferroelectric inclusion via the minimization of the modified thermodynamic potential $\widetilde{G}$ given by Eq. (9).

(iv) Solve the system of nine simultaneous Eqs. (25) and (26) with the matrix parameters $S_{i j}^{0 m}, c_{i j k l}^{*}$, and $\varepsilon_{i j}^{*}$ set equal to the above approximate values, basic matrices $B_{k l m n}$ determined as described in (ii), and some reasonable values chosen for the measuring external fields $E_{n}^{a}$ and $S_{m n}^{a}$. A set of solutions $S_{u v}^{0 x}$ and $P_{i}^{0 x}$ must 
be obtained, corresponding to various possible lattice orientations in a representative crystallite relative to the fixed reference frame $(x, y, z)$.

(v) Calculate auxiliary quantities $\Delta \sigma_{i j}^{*}, \Delta P_{n}^{*}$, and $\Delta E_{n}^{*}$ from Eqs. (11)-(14) using the values of $S_{u v}^{0 x}$ and $P_{i}^{0 x}$ obtained in (iv). Then determine via Eqs. (15), (16), and (18) the stresses $\Delta \sigma_{i j}(\varphi, \psi, \theta)$ and electric induction $\Delta D_{n}(\varphi, \psi, \theta)$ inside a ferroelectric crystallite as a function of the lattice orientation for given applied fields $E_{n}^{a}$ and $S_{m n}^{a}$. Using Eqs. (27)-(29), calculate the mean values $\left\langle S_{m n}^{g}\right\rangle,\left\langle\Delta \sigma_{i j}\right\rangle,\left\langle\Delta D_{n}\right\rangle$ of the total strain $S_{m n}^{g}$ and the stress and induction changes $\left(\Delta \sigma_{i j}\right.$ and $\left.\Delta D_{n}\right)$ in an ensemble of crystallites. Then determine the dependences of $\left\langle\Delta \sigma_{i j}\right\rangle$ and $\left\langle\Delta D_{n}\right\rangle$ on applied fields and evaluate the small-signal aggregate material constants $\left\langle c_{i j m n}\right\rangle$ and $\left\langle\varepsilon_{i n}\right\rangle$ from the slopes of these dependences at $S_{m n}^{a} \rightarrow 0$ and $E_{n}^{a} \rightarrow 0$ in accordance with Eqs. (30) and (31).

(vi) Repeat the sequence of operations (ii)-(v) with the matrix characteristics $S_{i j}^{0 m}, c_{i j k l}^{*}$, and $\varepsilon_{i j}^{*}$ set equal to the above values of $\left\langle S_{i j}^{g}\right\rangle,\left\langle c_{i j k l}\right\rangle$, and $\left\langle\varepsilon_{i j}\right\rangle$ instead of the quantities calculated in (i). Use the obtained new set of parameters $\left\langle S_{i j}^{g}\right\rangle,\left\langle c_{i j k l}\right\rangle,\left\langle\varepsilon_{i j}\right\rangle$ as the next approximation for the matrix characteristics. Continue the calculations until the difference between two successive estimates of the aggregate material constants becomes negligible. This self-consistent result describes the sought elastic and dielectric constants of a ferroelectric ceramic.

\section{NUMERICAL RESULTS FOR NANOCRYSTALLINE $\mathrm{Pb}\left(\mathrm{Zr}_{1-\chi} \mathrm{Ti}_{X}\right) \mathrm{O}_{3}$ AND BaTiO ${ }_{3}$ CERAMICS}

We performed numerical calculations necessary to determine the actual phase states and dielectric properties of two ferroelectric ceramics-lead zirconate titanate $\mathrm{Pb}\left(\mathrm{Zr}_{1-x} \mathrm{Ti}_{x}\right) \mathrm{O}_{3}(\mathrm{PZT})$ and barium titanate $\mathrm{BaTiO}_{3}(\mathrm{BT})$. The grains of these ceramics were assumed to be in a singledomain state, which is typical of small-size crystallites. The critical grain size $g^{*}$ in a ferroelectric polycrystal, below which the single-domain state is energetically favored over polydomain ones, can be evaluated from the comparison of the elastic energy reduction due to the formation of non- $180^{\circ}$ domain walls and the self-energy of these walls. In Ref. 22, it was shown that $g^{*} \approx 4 \pi^{2}(1-\nu) \gamma / \mu S_{0}^{2}$, where $\mu$ and $\nu$ are the effective shear modulus and Poisson's ratio of a polycrystalline material, $S_{0}$ is the characteristic spontaneous strain of a ferroelectric, and $\gamma$ is the self-energy per unit area of a domain wall. For $\mathrm{PbTiO}_{3}$ ceramics, the critical grain size $g^{*}$ was estimated to be about $50 \mathrm{~nm}^{22}$ In the case of PZT, the critical size $g^{*}$ is expected to be of the same order of magnitude. A larger value of $g^{*} \approx 300 \mathrm{~nm}$ has been obtained for BT, using the numerical values of $\mu, \nu$, and $S_{0}$ given in literature $^{38,39}$ and analyzing the experimental data. ${ }^{40}$ The above results show that grains in nanocrystalline PZT and BT ceramics must be mostly free of non- $180^{\circ}$ domain walls.

For PZT solid solutions, the calculations were carried out using the dielectric stiffnesses $\alpha_{1}, \alpha_{i j}$, and $\alpha_{i j k}$ at constant

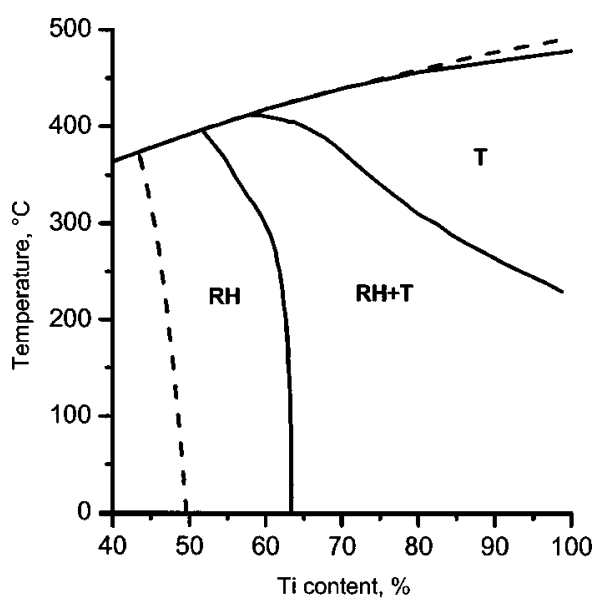

FIG. 1. Theoretical phase diagram of nanocrystalline $\mathrm{Pb}\left(\mathrm{Zr}_{1-x} \mathrm{Ti}_{x}\right) \mathrm{O}_{3}$ ceramics. Stability ranges of tetragonal and rhombohedral phases are denoted by the letters $\mathrm{T}$ and $\mathrm{RH}$, respectively. The region of the coexistence of tetragonal and rhombohedral crystallites is labeled as RH+T. Dashed lines show the morphotropic phase boundary and the line of ferroelectric phase transition calculated for coarse-grained $\mathrm{Pb}\left(\mathrm{Zr}_{1-x} \mathrm{Ti}_{x}\right) \mathrm{O}_{3}$ ceramics, where internal stresses are negligible due to the twinning of crystallites. This calculation was performed using Eq. (4) for the Gibbs free energy $G$ with the involved thermodynamic parameters taken from Ref. 33 .

stress and the electrostrictive constants $Q_{i j k l}$ taken from Ref. 33 and the elastic compliances $s_{i j k l}^{P}$ at constant polarization given in Ref. 41. (All these parameters were assumed to be independent of temperature, ${ }^{33,41}$ except for the dielectric stiffness $\alpha_{1}$ which was given a linear temperature dependence based on the Curie-Weiss law.) To describe the effect of composition on the properties of PZT ceramics, these material constants were calculated as continuous functions of the Ti content $x$ from the available discrete sets ${ }^{33,41}$ by means of spline interpolation. The theoretical analysis was restricted by compositions $x \geqslant 0.4$ and temperatures above $T=0{ }^{\circ} \mathrm{C}$ to ensure good accuracy of the approximation (4) used for the Gibbs function $G_{0}\left(P_{i}\right)^{41}$

Figure 1 shows the phase diagram of nanocrystalline PZT ceramics that results from our numerical calculations. Depending on the Ti content and temperature, the crystallites in these ceramics were found to stabilize either in a tetragonal or in a rhombohedral phase, which corresponds to the crystal structures of conventional PZT ceramics. ${ }^{42}$ However, there is a drastic difference from the behavior of polycrystals with twinned grains. Indeed, in a wide range of compositions and temperatures, tetragonal crystallites coexist with the rhombohedral ones in a nanocrystalline ceramic with the given $\mathrm{Zr} / \mathrm{Ti}$ ratio. This remarkable theoretical result was obtained somewhat unexpectedly during the numerical calculations. It was initially found that the procedure described in Sec. III does not give any self-consistent solution of the problem in some region of the $(x, T)$ plane. In order to overcome this difficulty, we supposed that a mixture of tetragonal and rhombohedral crystallites may represent the energetically most favorable phase state of a nanocrystalline ceramic in this region.

To check this supposition, we modified the aforementioned procedure by introducing two inclusion/matrix systems, which differ by the phase state of a ferroelectric inclusion (phase I and phase II) embedded into the same matrix. 
The polarization components $P_{i}^{g \mathrm{I}}$ and $P_{i}^{g \mathrm{II}}$ and total strains $S_{m n}^{g \mathrm{I}}$ and $S_{m n}^{g \mathrm{II}}$ in these inclusions can be evaluated by finding the minima $\widetilde{G}^{\mathrm{I}}$ and $\widetilde{G}^{\mathrm{II}}$ (absolute and relative) of the modified thermodynamic potential $\widetilde{G}$ given by Eq. (9). On each iteration, the energetically most favorable aggregate state is determined via the minimization of the mean thermodynamic potential

$$
\langle\widetilde{G}\rangle=q \widetilde{G}^{\mathrm{I}}+(1-q) \widetilde{G}^{\mathrm{II}},
$$

with respect to the volume fraction $q$ of phase I in a polycrystal. The mean values $\left\langle S_{m n}^{g}\right\rangle,\left\langle\Delta \sigma_{i j}\right\rangle$, and $\left\langle\Delta D_{n}\right\rangle$ of the total strain and the field-induced stress and induction changes in a mixture of crystallites are calculated as

$$
\begin{aligned}
& \left\langle S_{m n}^{g}\right\rangle=q^{*}\left\langle S_{m n}^{g \mathrm{I}}\right\rangle+\left(1-q^{*}\right)\left\langle S_{m n}^{g \mathrm{II}}\right\rangle, \\
& \left\langle\Delta \sigma_{i j}\right\rangle=q^{*}\left\langle\Delta \sigma_{i j}^{\mathrm{I}}\right\rangle+\left(1-q^{*}\right)\left\langle\Delta \sigma_{i j}^{\mathrm{II}}\right\rangle, \\
& \left\langle\Delta D_{n}\right\rangle=q^{*}\left\langle\Delta D_{n}^{\mathrm{I}}\right\rangle+\left(1-q^{*}\right)\left\langle\Delta D_{n}^{\mathrm{II}}\right\rangle,
\end{aligned}
$$

where $q^{*}$ is the optimum volume fraction of phase I, and $\left\langle S_{m n}^{g \mathrm{I}}\right\rangle,\left\langle\Delta \sigma_{i j}^{\mathrm{I}}\right\rangle$, and $\left\langle\Delta D_{n}^{\mathrm{I}}\right\rangle$ and $\left\langle S_{m n}^{g \mathrm{II}}\right\rangle,\left\langle\Delta \sigma_{i j}^{\mathrm{II}}\right\rangle$, and $\left\langle\Delta D_{n}^{\mathrm{II}}\right\rangle$ are computed via Eqs. (27)-(29) for the inclusions with the phase states I and II, respectively. The small-signal aggregate material constants $\left\langle c_{i j m n}\right\rangle$ and $\left\langle\varepsilon_{i n}\right\rangle$ can be evaluated as explained in procedure (v) above and then used to find the ceramic elastic and dielectric constants with the aid of an iterative procedure described in procedure (vi).

Our numerical calculations confirmed that the mixture of tetragonal and rhombohedral crystallites represents the most favorable state of nanocrystalline PZT ceramics in a large section of the phase diagram (Fig. 1). This region of phase coexistence, $R_{r+t}$, may be regarded as a substitute for the morphotropic phase boundary (MPB) existing in conventional PZT. ${ }^{42}$ However, the left boundary of $R_{r+t}$ is significantly shifted from the MPB to larger Ti contents $x$ (by $\Delta x$ $=10 \%-15 \%$; see Fig. 1). The right boundary is situated at temperatures larger than $200{ }^{\circ} \mathrm{C}$ so that even at $x=100 \%$ the rhombohedral phase should exist at room temperature in some grains of a nanocrystalline ceramic. We recall that, in conventional form, $\mathrm{PbTiO}_{3}$ remains tetragonal at any temperature below the ferroelectric transition. ${ }^{34}$

Strong enlargement of the rhombohedral field in the phase diagram of nanocrystalline PZT ceramics is evidently caused by the lack of stress relaxation via the twinning. The mechanical stresses, arising in crystallites due to elastic clamping by the surrounding material, favor the formation of rhombohedral phase. In addition, when the twinning is not allowed, the mixing of tetragonal and rhombohedral crystallites represents an effective channel for the stress relaxation. It should be noted that the coexistence of tetragonal and rhombohedral phases was experimentally observed in epitaxial PZT thin films. ${ }^{43}$

The calculations also demonstrated that the paraelectricto-ferroelectric phase transition in nanocrystalline PZT ceramics is always of the second order, irrespective of the $\mathrm{Zr} / \mathrm{Ti}$ ratio. This is different from the behavior of conventional PZT, where the first-order phase transition takes place at $x \geqslant 80 \% .^{33}$ The order of ferroelectric phase transition

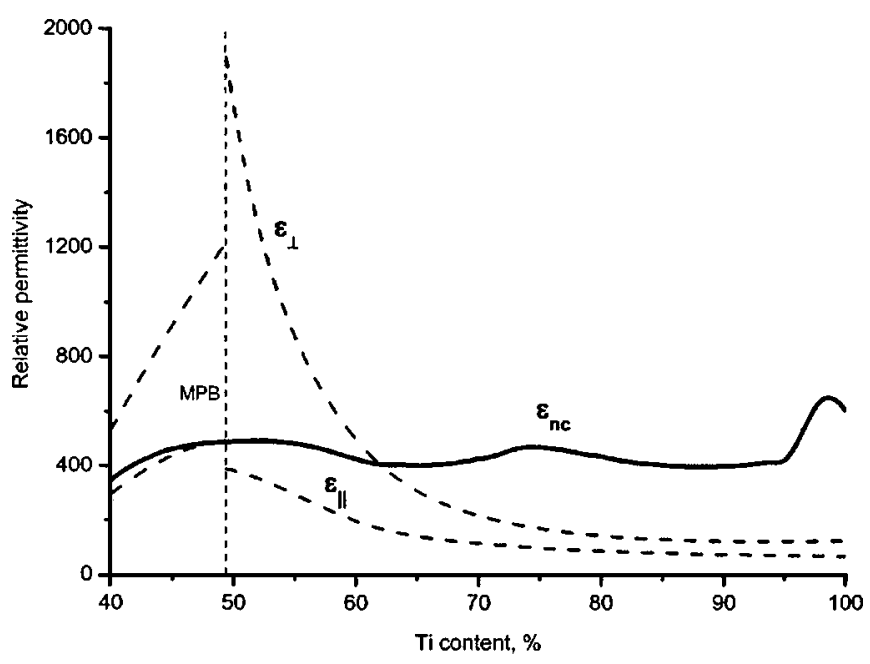

FIG. 2. Dielectric response $\varepsilon_{\mathrm{nc}}$ of a nanocrystalline $\mathrm{Pb}\left(\mathrm{Zr}_{1-x} \mathrm{Ti}_{x}\right) \mathrm{O}_{3}$ ceramic (solid line) in comparison with the permittivities $\varepsilon_{\|}$and $\varepsilon_{\perp}$ of a singledomain stress-free crystallite (dashed lines). The temperature is taken to be $25^{\circ} \mathrm{C}$. The permittivities $\varepsilon_{\|}$and $\varepsilon_{\perp}$ in the directions parallel and perpendicular to the polarization vector were calculated from the theoretical thermodynamic parameters of $\mathrm{Pb}\left(\mathrm{Zr}_{1-x} \mathrm{Ti}_{x}\right) \mathrm{O}_{3}$ given in Ref. 33 .

changes due to the three-dimensional elastic clamping of single-domain crystallites in a nanocrystalline ceramic. The analysis shows that this clamping renormalizes the fourthorder polarization term in the thermodynamic potential. ${ }^{22}$ At $x \geqslant 80 \%$, the renormalization changes the sign of the fourthorder coefficient $\alpha_{11}$ from negative ${ }^{33}$ to positive.

In addition to the phase diagram, we calculated numerically the macroscopic dielectric response $\varepsilon_{\mathrm{nc}}$ of an unpolarized nanocrystalline PZT ceramic as a function of composition and temperature. Figure 2 shows the dependence of small-signal permittivity $\varepsilon_{\mathrm{nc}}$ on the Ti content at room temperature. It can be seen that the permittivity varies nonmonotonically with the composition of solid solution, reaching its maximum value of $\varepsilon_{\mathrm{nc}} \approx 640$ at the Ti content $x \approx 98.5 \%$. However, there is no dielectric anomaly at the boundary between the rhombohedral field and the region of phase coexistence $R_{r+t}$ in the stability diagram.

For the better understanding of the above results, we also calculated the theoretical dielectric responses of individual PZT crystallites. Assuming that the crystallite is free of internal stresses and domain walls, we found the permittivities $\varepsilon_{\|}$and $\varepsilon_{\perp}$ in the directions parallel and perpendicular to the spontaneous polarization to vary with the $\mathrm{Ti}$ content as shown in Fig. 2. Although the transverse dielectric response $\varepsilon_{\perp}$ increases drastically near the MPB, the permittivity $\varepsilon_{\mathrm{nc}}$ of nanocrystalline PZT remains moderate in this range of compositions, showing only a weak broad maximum around $x$ $=50 \%$. On the other hand, $\varepsilon_{\mathrm{nc}}$ appears to be about three times higher than the single-crystal responses $\varepsilon_{\|}$and $\varepsilon_{\perp}$ at $x$ $\geqslant 75 \%$. This result demonstrates that the elastic clamping may strongly increase the intrinsic dielectric response of a ferroelectric crystallite.

For nanocrystalline BT ceramics, numerical calculations were performed using the dielectric stiffnesses $\alpha_{1}, \alpha_{i j}$, and $\alpha_{i j k}$, electrostrictive constants $Q_{i j k l}$, and elastic compliances $s_{i j k l}^{P}$ listed in Ref. 44. (The stiffnesses $\alpha_{1}, \alpha_{11}$, and $\alpha_{111}$ of BT linearly depend on temperature. ${ }^{44}$ ) Depending on tempera- 


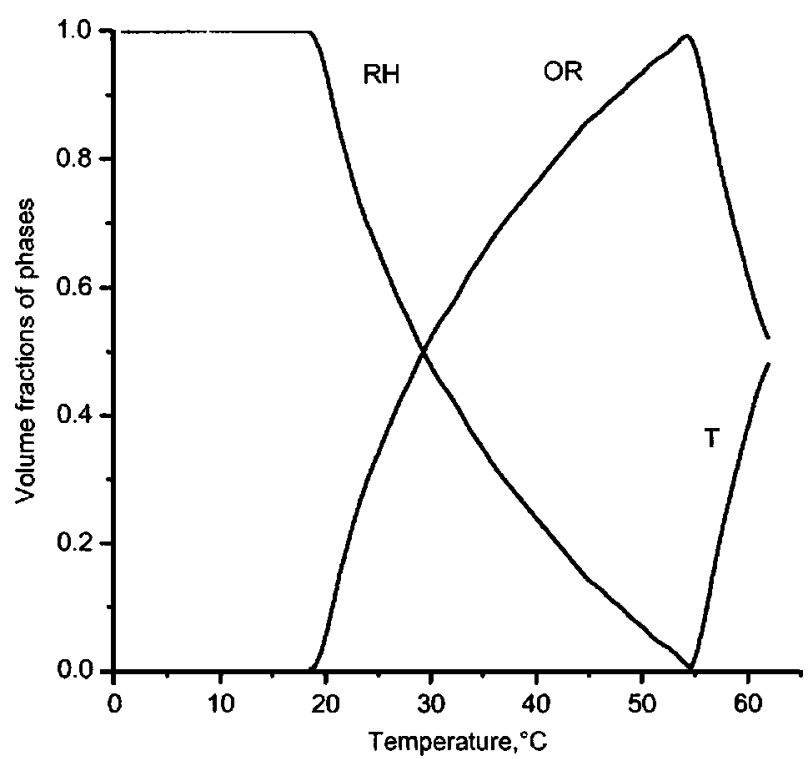

FIG. 3. Equilibrium volume fractions of the rhombohedral (RH), orthorhombic (OR), and tetragonal (T) phases in nanocrystalline $\mathrm{BaTiO}_{3}$ ceramics as a function of temperature.

ture, the clamped single-domain ferroelectric crystallites were found to stabilize in the rhombohedral, orthorhombic, or tetragonal state. The low-temperature rhombohedral phase represents the energetically most favorable state up to $19{ }^{\circ} \mathrm{C}$ in contrast to the stress-free single crystal, where this phase is stable only below $-71{ }^{\circ} \mathrm{C} .{ }^{32}$ Moreover, at temperatures ranging from 19 to $55^{\circ} \mathrm{C}$, the nanocrystalline BT ceramic contains a mixture of rhombohedral and orthorhombic crystallites. The volume fraction of rhombohedral phase gradually decreases with increasing temperature in this range, whereas the fraction of orthorhombic crystallites increases accordingly (see Fig. 3). Just above $T \approx 55^{\circ} \mathrm{C}$, the structure of nanocrystalline ceramic transforms into the mixture of orthorhombic and tetragonal crystallites. Unfortunately, at temperatures above $60{ }^{\circ} \mathrm{C}$, it becomes impossible to find a self-consistent solution for the ceramic properties with the aid of our iterative procedure. Nevertheless, the above results clearly demonstrate that, in the most important temperature range around $20^{\circ} \mathrm{C}$, the phase state of a nanocrystalline BT ceramic differs drastically from that of a single crystal, where the tetragonal phase is stable above $10{ }^{\circ} \mathrm{C}$. $^{32}$

Our theoretical predictions may be compared with the experimental data obtained for nanocrystalline BT ceramics by Frey and Payne. ${ }^{11}$ Using high-resolution scanning electron microscopy, these authors found that BT ceramics with the grain size $g \leqslant 100 \mathrm{~nm}$ are free of non- $180^{\circ}$ domains (twins), whereas at $g=400 \mathrm{~nm}$ some grains are twinned. These observations support our theoretical estimate $g^{*}$ $\approx 300 \mathrm{~nm}$ of the critical grain size in BT polycrystals. The $\mathrm{X}$-ray diffraction (XRD) investigations, which were based on the examination of the $\{200\}$ pseudocubic reflections, showed the absence of tetragonal lattice distortions in ceramics with grain sizes below $100 \mathrm{~nm}$. The Raman-scattering data, however, indicated the existence of orthorhombic structure in these ceramics at room temperature rather than the cubic one, which agrees with one of our predictions. The presence of additional rhombohedral crystallites here also

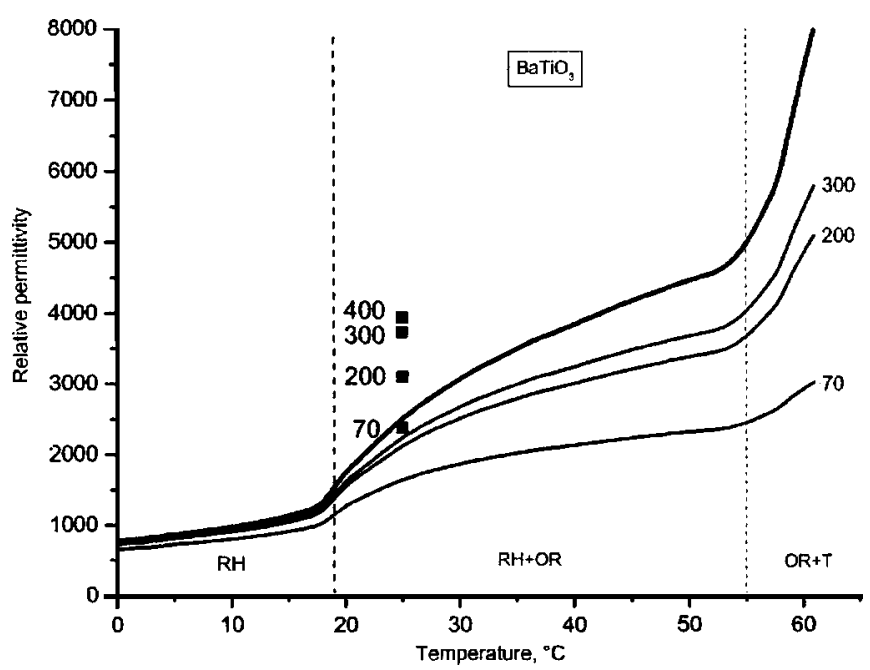

FIG. 4. Temperature dependence of permittivity calculated for nanocrystalline $\mathrm{BaTiO}_{3}$ ceramics of different grain sizes. The upper curve corresponds to a perfect ceramic, where grain boundaries do not suppress the macroscopic dielectric response. Three other curves describe the grain-size effect on the permittivity $\varepsilon_{\mathrm{nc}}^{d}$ of a ceramic with structurally disordered layers between crystallites. Squares show the experimental data obtained for $\mathrm{BaTiO}_{3}$ ceramics in Ref. 47. Numbers indicate the grain size in nanometers. The predicted crystal structure of a nanocrystalline $\mathrm{BaTiO}_{3}$ ceramic is denoted by RH (rhombohedral phase), RH+OR (mixture of rhombohedral and orthorhombic crystallites), and $\mathrm{OR}+\mathrm{T}$ (mixture of orthorhombic and tetragonal crystallites).

cannot be ruled out because the formation of the rhombohedral phase does not lead to the splitting of the $\{200\}$ reflections. $^{45}$

For the macroscopic dielectric response of unpolarized nanocrystalline BT ceramics, we obtained the results presented in Fig. 4. In contrast to the dielectric behavior of BT single crystals, ${ }^{32}$ the permittivity varies monotonically with increasing temperature. Since the grain boundaries are known to reduce the measured permittivity of BT ceramics, ${ }^{46-49}$ we estimated the grain-boundary effect on the dielectric response in addition to its temperature dependence. Assuming that grain boundaries have a low permittivity $\varepsilon_{d}$ and an effective thickness $d$, the aggregate macroscopic response $\varepsilon_{\mathrm{nc}}^{d}$ of a polycrystal can be evaluated as

$$
\varepsilon_{\mathrm{nc}}^{d}(g) \approx \frac{3 \varepsilon_{d}\left(\varepsilon_{\mathrm{nc}}+2 \varepsilon_{d}\right)}{\varepsilon_{\mathrm{nc}}+2 \varepsilon_{d}-\left(\varepsilon_{\mathrm{nc}}-\varepsilon_{d}\right)\left(1-\frac{2 d}{g}\right)^{3}}-2 \varepsilon_{d}
$$

where $\varepsilon_{\mathrm{nc}}$ is the permittivity of a perfect nanocrystalline ceramic having $d=0$. Equation (36) is based on the relation derived in Ref. 49 and on the assumption that the average permittivity of the grain interior may be approximated by $\varepsilon_{\mathrm{nc}}$. Calculating the temperature dependence of $\varepsilon_{\mathrm{nc}}$ numerically and using the values $\varepsilon_{d}=100$ and $d=0.7 \mathrm{~nm}$ given in Ref. 49, we obtained the set of curves plotted in Fig. 4. At temperatures below $19{ }^{\circ} \mathrm{C}$, where nanocrystalline BT ceramics stabilize in the rhombohedral phase, the grain-boundary effect on the macroscopic dielectric response $\varepsilon_{\mathrm{nc}}^{d}$ is relatively small. With the increase of temperature, however, the suppression of $\varepsilon_{\mathrm{nc}}^{d}$ by the low-permittivity grain boundaries intensifies. At $T \approx 50^{\circ} \mathrm{C}$ and $g=70 \mathrm{~nm}$, for instance, $\varepsilon_{\mathrm{nc}}^{d}$ be- 


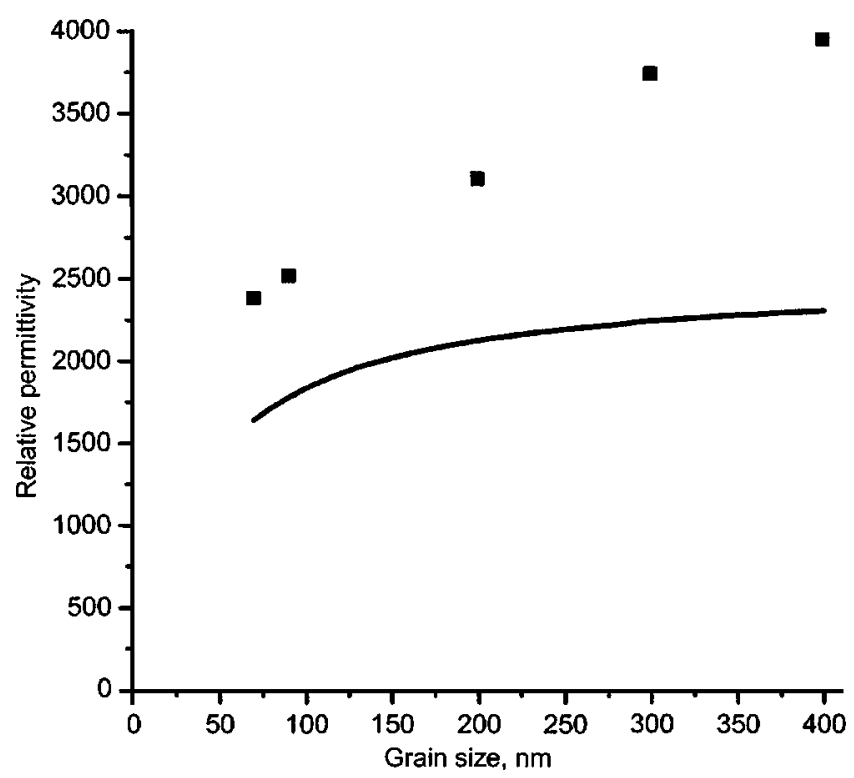

FIG. 5. Room-temperature dielectric response of a nanocrystalline $\mathrm{BaTiO}_{3}$ ceramic as a function of the grain size. Solid line shows the theoretical dependence, whereas squares denote the experimental values given in Ref. 47.

comes about two times smaller than the "perfect" permittivity $\varepsilon_{\mathrm{nc}}$.

Our theoretical results may be compared with the measured dielectric properties of nanocrystalline BT ceramics. $^{47,48}$ At the minimum studied grain size $g=40 \mathrm{~nm}$, the room-temperature dielectric response was found to be about $900 .{ }^{47}$ The corresponding theoretical value of $\varepsilon_{\mathrm{nc}}^{d}$ $\approx 1300$ is in reasonable agreement with the measured permittivity. The predicted grain-size dependence of the dielectric response shows the same trend as the observed one (see Fig. 5), but the difference between the theoretical and experimental values becomes larger with increasing grain size. There are several possible reasons which may explain why the theory underestimates the ceramic permittivity. We believe that the inhomogeneity of internal stresses inside real grains of irregular shape, which was neglected in our model, may result in a higher measured dielectric response.

\section{CONCLUSIONS}

(1) In nanocrystalline ferroelectric ceramics, the equilibrium phase states of elastically clamped crystallites may differ drastically from those of single crystals and coarsegrained materials. This is due to the lack of stress relaxation via domain formation (twinning), which becomes energetically unfavorable below some critical grain size.

(2) The phase diagram of nanocrystalline $\mathrm{Pb}\left(\mathrm{Zr}_{1-x} \mathrm{Ti}_{x}\right) \mathrm{O}_{3}$ ceramics is distinguished by the presence of a wide range of compositions and temperatures, where a mixture of rhombohedral and tetragonal crystallites represents the energetically most favorable thermodynamic state. This region of phase coexistence substitutes the morphotropic phase boundary characteristic of conventional $\mathrm{Pb}\left(\mathrm{Zr}_{1-x} \mathrm{Ti}_{x}\right) \mathrm{O}_{3}$ ceramics.

(3) In nanocrystalline $\mathrm{BaTiO}_{3}$ ceramics, the stability range of the low-temperature rhombohedral phase extends up to about $19^{\circ} \mathrm{C}$. Above this temperature, ceramics contain a mixture of rhombohedral and orthorhombic crystallites, which transforms into a mixture of orthorhombic and tetragonal crystallites at about $55^{\circ} \mathrm{C}$.

(4) The dielectric properties of nanocrystalline ferroelectric ceramics may differ markedly from those of conventional materials owing to the elastic clamping of singledomain crystallites. In the case of $\mathrm{Pb}\left(\mathrm{Zr}_{1-x} \mathrm{Ti}_{x}\right) \mathrm{O}_{3}$ ceramics with $x \geqslant 0.75$, the mechanical grain/grain interaction strongly increases the intrinsic dielectric response of ferroelectric crystallites.

\section{ACKNOWLEDGMENT}

The research described in this publication was made possible in part by Grant No. I/75965 from the VolkswagenStiftung, Germany.

${ }^{1}$ N. Setter and R. Waser, Acta Mater. 48, 151 (2000).

${ }^{2}$ H. Kniepkamp and W. Heywang, Z. Angew. Phys. 9, 385 (1954).

${ }^{3}$ K. Kinoshita and A. Yamaji, J. Appl. Phys. 47, 371 (1976).

${ }^{4}$ G. Arlt, D. Hennings, and G. de With, J. Appl. Phys. 58, 1619 (1985).

${ }^{5}$ G. Arlt, J. Mater. Sci. 25, 2655 (1990).

${ }^{6}$ M. P. McNeal, S.-J. Jang, and R. E. Newnham, J. Appl. Phys. 83, 3288 (1998).

${ }^{7}$ K. Ishikawa, K. Yoshikawa, and N. Okada, Phys. Rev. B 37, 5852 (1988).

${ }^{8}$ K. Uchino, E. Sadanaga, and T. Hirose, J. Am. Ceram. Soc. 72, 1555 (1989).

${ }^{9}$ M. de Keijser, G. J. M. Dormans, P. J. van Veldhoven, and D. M. de Leeuw, Appl. Phys. Lett. 59, 3556 (1991).

${ }^{10}$ M. H. Frey and D. A. Payne, Appl. Phys. Lett. 63, 2753 (1993).

${ }^{11}$ M. H. Frey and D. A. Payne, Phys. Rev. B 54, 3158 (1996).

${ }^{12}$ H.-I. Hsiang and F.-S. Yen, J. Am. Ceram. Soc. 79, 1053 (1996).

${ }^{13}$ L. A. Bursill, B. Jiang, J. L. Peng, T. L. Ren, W. L. Zhong, and P. L. Zhang, Ferroelectrics 191, 281 (1997).

${ }^{14}$ R. Kretschmer and K. Binder, Phys. Rev. B 20,1065 (1979).

${ }^{15}$ S. Li, J. A. Eastman, Z. Li, C. M. Foster, R. E. Newnham, and L. E. Cross, Phys. Lett. A 212, 341 (1996).

${ }^{16} \mathrm{~S}$. Li, J. A. Eastman, J. M. Vetrone, C. M. Foster, R. E. Newnham, and L. E. Cross, Jpn. J. Appl. Phys., Part 1 36, 5169 (1997).

${ }^{17}$ I. I. Ivanchik, Sov. Phys. Solid State 3, 2705 (1962).

${ }^{18}$ I. P. Batra and B. D. Silverman, Solid State Commun. 11, 291 (1972).

${ }^{19}$ H. Huang, C. Q. Sun, Z. Tianshu, and P. Hing, Phys. Rev. B 63, 184112 (2001).

${ }^{20}$ W. R. Buessem, L. E. Cross, and A. K. Goswami, J. Am. Ceram. Soc. 49, 33 (1966).

${ }^{21}$ E. M. Pikalev and V. I. Aleshin, Sov. Phys. Solid State 31, 2079 (1989).

${ }^{22}$ N. A. Pertsev and E. K. H. Salje, Phys. Rev. B 61, 902 (2000).

${ }^{23}$ M. Marutake, J. Phys. Soc. Jpn. 11, 807 (1956).

${ }^{24}$ A. V. Turik and A. I. Chernobabov, Sov. Phys. Tech. Phys. 22, 1127 (1977).

${ }^{25}$ V. I. Aleshin, Kristallografiya 32, 422 (1987).

${ }^{26}$ V. I. Aleshin and E. M. Pikalev, Zh. Tekh. Fiz. 60, 129 (1990).

${ }^{27}$ T. Olson and M. Avellaneda, J. Appl. Phys. 71, 4455 (1992)

${ }^{28}$ M. L. Dunn, J. Appl. Phys. 78, 1533 (1995).

${ }^{29}$ C.-W. Nan and D. R. Clarke, J. Am. Ceram. Soc. 79, 2563 (1996).

${ }^{30}$ N. A. Pertsev, A. G. Zembilgotov, and R. Waser, J. Appl. Phys. 84, 1524 (1998).

${ }^{31}$ A. F. Devonshire, Philos. Mag. 40, 1040 (1949).

${ }^{32}$ F. Jona and G. Shirane, Ferroelectric Crystals (Macmillan, New York, 1962).

${ }^{33}$ M. J. Haun, E. Furman, S. J. Jang, and L. E. Cross, Ferroelectrics 99, 13 (1989).

${ }^{34}$ M. J. Haun, E. Furman, S. J. Jang, H. A. McKinstry, and L. E. Cross, J. Appl. Phys. 62, 3331 (1987).

${ }^{35}$ J. D. Eshelby, Proc. R. Soc. London, Ser. A 241, 376 (1957).

${ }^{36}$ B. Wang, Int. J. Solids Struct. 29, 293 (1992).

${ }^{37}$ Y. Benveniste, J. Appl. Phys. 72, 1086 (1992).

${ }^{38}$ A. V. Turik, Sov. Phys. Solid State 12, 688 (1970). 
${ }^{39}$ N. A. Pertsev and G. Arlt, Ferroelectrics 123, 27 (1991).

${ }^{40}$ To estimate the critical grain size $g^{*}$ for $\mathrm{BaTiO}_{3}$, we also assumed that the $90^{\circ}$ domain-wall energy $\gamma$ in this ferroelectric is about $0.03 \mathrm{~J} / \mathrm{m}^{2}$. This value was obtained by comparing the theoretical grain-size dependence of the domain width, which was derived in Ref. 39, with the experimental data given in Ref. 5. The least-square fitting of these data with varying parameter $\gamma$ shows that the best agreement is achieved at $\gamma=0.03 \mathrm{~J} / \mathrm{m}^{2}$.

${ }^{41}$ N. A. Pertsev, V. G. Kukhar, H. Kohlstedt, and R. Waser, Phys. Rev. B 67, 054107 (2003)

${ }^{42}$ B. Jaffe, W. R. Cook, and H. Jaffe, Piezoelectric Ceramics (Academic, London, 1971).

${ }^{43}$ S. H. Oh and H. M. Jang, Phys. Rev. B 63, 132101 (2001).

${ }^{44}$ N. A. Pertsev, A. G. Zembilgotov, and A. K. Tagantsev, Ferroelectrics
223, 79 (1999).

${ }^{45}$ It should be noted that the lattice structure of extremely small crystallites may differ from that predicted by our theory. This is due to the fact that we neglected the presence of surface layers with altered physical properties in ferroelectric crystallites and the influence of these layers on the interior.

${ }^{46}$ D. A. Payne and L. E. Cross, in Microstructure and Properties of Ceramic Materials, edited by T. S. Yen and J. A. Pask (Science, Beijing, 1984).

${ }^{47}$ M. N. Frey, Ph.D. thesis, University of Illinois at Urbana-Champaign, 1996 (unpublished).

${ }^{48}$ M. H. Frey, Z. Xu, P. Han, and D. A. Payne, Ferroelectrics 206-207, 337 (1998).

${ }^{49}$ A. Yu. Emelyanov, N. A. Pertsev, S. Hoffmann-Eifert, U. Böttger, and R. Waser, J. Electroceram. 9, 5 (2002). 